\title{
VARIAÇÃO ESPAÇO-TEMPORAL DA CPUE PARA O GÊNERO RHINOBATOS (RAJIFORMES, RHINOBATIDAE) NA COSTA SUDESTE E SUL DO BRASIL
}

\author{
MARTINS, R.R. \& P.R. SCHWINGEL \\ Centro de Educação Superior em Ciências Tecnológicas, Da terra e Do Mar \\ Universidade do Vale do Itajaí/UNIVALI C.P. 360-Itajaí-Brasil - CEP 88302-202 \\ kekarenno@terra.com.br
}

RESUMO

\begin{abstract}
Na costa sudeste e sul do Brasil, ocorrem duas espécies do gênero Rhinobatos, i.e Rhinobatos percellens e $R$. horkelli, estas fazem parte das capturas da pesca multiespecífica das frotas de arrasto duplo, parelha e simples, sendo que a captura da raia-viola vem diminuindo crescentemente desde a última década, juntamente com sua CPUE. Este trabalho analisou a variação espaço-temporal da CPUE da raia-viola na costa sudeste e sul do Brasil, através do cálculo da CPUE mensal e análise da distribuição das capturas realizadas pela frota arrasteira que desembarcou no estado de Santa Catarina entre 2000 e 2002. Neste período as capturas de raiaviola se concentraram entre Imbituba - SC e Chuí - RS. Um padrão de variação espaço-temporal das capturas não foi observado, entretanto, ocorreram picos de primavera e verão para capturas com arrasto duplo e de parelhas em 2001 e 2002. No período de estudo, verificou-se um incremento da CPUE para a raia-viola em comparação à década de 1990, o que pode ser um indicador do aumento de sua disponibilidade. A frota de arrasto de parelha atuou principalmente em profundidades inferiores a $100 \mathrm{~m}$, enquanto as frotas de arrasto duplo e simples operaram, a partir de 2001, em maiores profundidades $(>100 \mathrm{~m})$, alcançando CPUE mais elevadas. Este deslocamento das frotas de arrasto para regiões mais profundas, explicam o aumento da CPUE para a raia-viola observado no período de 2000 a 2002.
\end{abstract}

Palavras-chave: Rhinobatos, CPUE, frotas de arrasto, variação espaço-temporal.

\section{SPATIAL - TEMPORAL VARIATIONS OF CPUE OF GENUS RHINOBATOS (RAJIFORMES, RHINOBATIDAE) IN SOUTHEASTERN AND SOUTHERN COAST OF BRAZIL}

\begin{abstract}
Two species of guitarfish, Rhinobatos percellens and R. horkelli, are found off southeastern and southern coast of Brazil. Both are components of a multiespecific fishery of double-rig, pair and single trawl. Catches and CPUE of guitarfish were decreasing sharply during last decade. This present work analyzed the spatial-temporal variations of catch per unit effort of guitarfish off southeastern and southern coast of Brazil, through analysis of CPUE data from trawlers fishery, which landed in state of Santa Catarina, between 2000 and 2002. The guitarfish catches was concentrated between Imbituba-SC and Chuí-RS. A pattern of spatial-temporal variations of catches have not been observed, however, did occur spring and summer high to pair and double-rigs trawlers catches. In the period of study was observed an improvement of guitarfish CPUE that could be a sign of disponibylity increase. The pair trawlers actuated principally in lower than $100 \mathrm{~m}$ deeps, in spit of double-rig and other trawler operated, from 2001, in high deeps reaching bigger CPUE. These displacements of trawlers to deeper water explain the increment of guitarfish CPUE that was observed between 2000 and 2002.
\end{abstract}

Key-words: Rhinobatos, CPUE, trawlers, spatial-temporal variations 


\section{INTRODUÇÃO}

A família Rhinobatidae (raias-viola) é composta por 7 gêneros que se distribuem pelos Oceanos Atlântico, Indico, Pacífico e no mar Mediterrâneo. No Brasil, ocorrem dois gêneros, i.e. Rhinobatose Zapteryz. Duas espécies pertencentes ao gênero Rhinobatos ocorrem na costa sudeste e sul brasileira (Vooren, 1998), Rhinobatos horkelli (Müller \& Henle, 1841) e Rhinobatos percellens (Walbaum, 1792). Essas raias podem alcançar até $1,5 \mathrm{~m}$ de comprimento e chegam a pesar $12 \mathrm{Kg}$, são espécies vivíparas aplacentárias e produzem de 4 a 12 filhotes por gestação (Lessa et al., 1986).

Na costa sudeste e sul do Brasil, $R$. percellens e $R$. horkelli são componentes da captura realizada pela frota de arrasto (simples, duplo e parelhas) e emalhe de fundo (Mazzoleni \& Schwingel, 1999). Atualmente estão sediados em Santa Catarina 370 arrasteiros duplos, 59 parelhas e 37 arrasteiros simples, operando de forma multiespecífica, porém direcionada a algumas espécies-alvos (Perez et al., 2001).

A captura por unidade de esforço (CPUE) dos elasmobrânquios da pesca industrial está decrescendo desde 1985 e várias espécies vêm sofrendo redução de sua abundância (Vooren, 1998), principalmente devido ao fato de serem muito vulneráveis a alta pressão de pesca, pois apresentam maturação sexual tardia e baixa fecundidade. No Estado de Santa Catarina, os elasmobrânquios, no ano de 2000, representaram $6,5 \%$ da captura das frotas de arrasto, sendo relatado também que houve uma redução de $47 \%$ do peso total de peixes cartilaginosos desembarcados em 2000, em relação a 1998 (Pezzuto et al., 2001). Essa significativa queda de captura pode ser um indicador de sobrexplotação de várias espécies de elasmobrânquios. As sucessivas quedas de captura da raia-viola no Estado Rio Grande do Sul confirmam esse quadro. Entre 1975 e 1979, a captura média anual foi de $1010 \mathrm{t}$, entre 1980 e 1984, houve um acréscimo da média anual para 1253 t, entretanto, caiu para $901 \mathrm{t}$ entre 1985 e 1989. No período de 1990 até 1994, essa média caiu pela metade e chegou a $460 \mathrm{t}$ (Haimovici et al., 1998). Esse declínio das capturas, somados à queda da CPUE, que em 1984 era de 0,76 t/viagem e em 1992 passou para $0,05 \mathrm{t}$ /viagem, reforçam a tendência de que esta pescaria pode estar em colapso (Perez et al., 2001).

Diante desse quadro, o presente estudo tem como objetivo descrever a variação espaço-temporal da CPUE para raia-viola na costa sudeste e sul do Brasil, a fim de subsidiar o desenvolvimento de programas de manejo para este recurso pesqueiro.

\section{MATERIAL E MÉTODOS}

Para este estudo, foram utilizados dados das capturas realizadas pelas frotas de arrasto duplo, de parelhas e simples que desembarcaram nos portos de Itajaí e Navegantes (SC) entre os anos de 2000 e 2002. A coleta destes dados, fazem parte do programa de amostragem do CTTMar/UNIVALI. Esses dados são provenientes de Fichas de Produção e Entrevistas no Cais. As Fichas de Produção englobam dados de local e data de desembarques, bem como produção, em quilos, de cada categoria de pescado. A partir dos dados das fichas, foi calculada a capturas total de cada desembarque (i.e. viagem de pesca) e a captura da raia-viola, que inclui indiscriminadamente as duas espécies do gênero, $R$. percellens e $R$. horkelli. Das Entrevistas no Cais, analisouse dados de local e data do desembarque, áreas de pesca e profundidades da captura. Para um melhor entendimento das análises de distribuição sazonal e espacial das capturas, a plataforma sudeste e sul brasileira foi separada em zona rasa (até $100 \mathrm{~m}$ de profundidade), média (100 a 200 m) e profunda (acima de 200 $\mathrm{m})$.

A CPUE utilizada neste estudo tem como unidade de esforço, o número de viagens realizadas por cada modalidade de arrasto (du- 
plo, parelhas e simples) nos anos de 2000, 2001 e 2002. Como as viagens não foram direcionadas à captura de raia-viola, e a padronização dos esforços não foi realizada, a CPUE, neste caso, não pode ser um indicador de abundância deste recurso, mas sim de disponibilidade. Considerando que o coeficiente de capturabilidade é variável na pescaria da raiaviola devido a fatores como pesca não direcionada e migração da espécie, a CPUE está, portanto, associado à disponibilidade da raia-viola na área de pesca. A CPUE mensal foi calculada a partir da captura mensal total de raia-viola (t), em relação ao número total de viagens realizadas por mês por cada modalidade de arrasto. A CPUE anual foi calculada a partir da captura anual total de raia-viola (t), em relação ao número total de viagens realizadas por ano por cada frota de arrasto (simples, duplo e parelhas), separadamente.

\section{RESULTADOS E DISCUSSÃO}

\section{Variação sazonal da CPUE}

Para a frota de arrasto duplo, foram analisados 515 desembarques no ano de 2000, obtendo uma captura por unidade de esforço (CPUE) anual de raia-viola de $0,11 \mathrm{t}$ /viagem. A CPUE mensal variou de 0,015 a $0,43 \mathrm{t}$ por viagem (Fig. 1a; Tab. 1), sendo que os meses de abril e novembro atingiram os maiores índices, de 0,3 e 0,43 t/viagem, respectivamente. Para o ano de 2001, foram analisadas 612 viagens, resultando em uma CPUE anual de raia-viola de 0,11 t/viagem, verificando-se uma CPUE mensal entre 0,03 e 0,33 t/viagem (Fig. 1b; Tab. 1). Os meses de agosto e novembro foram os que alcançaram as maiores marcas. Durante o ano de 2002, dos arrasteiros duplos que desembarcaram em Santa Catarina, 499 viagens foram estudadas, apresentando uma CPUE anual de raia-viola na ordem de $0,15 \mathrm{t} / \mathrm{viagem}$, sendo que e a CPUE mensal variou entre 0,02 e 0,28 t/viagem (Fig. 1c; Tab. 1). Neste ano, as CPUE dos meses de maio e junho foram as mais expressivas $(0,26$ e $0,28 \mathrm{t} /$ viagem, respectivamente).

A frota de arrasto de parelha foi investigada baseando-se em 165 viagens du-
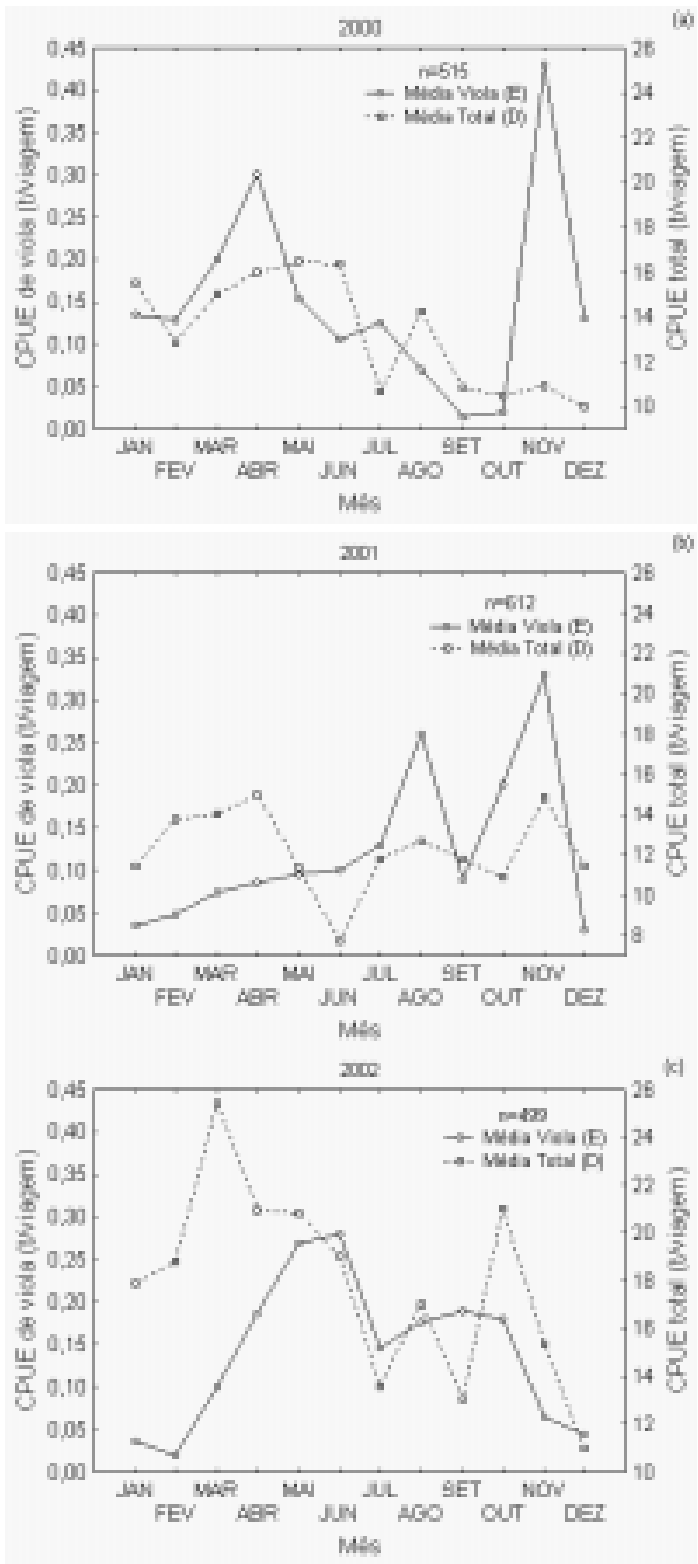

Figura 1a-c - Sazonalidade da CPUE da raia viola (Rhinobatos percellens e $R$. horkelli) pela frota de arrasto duplo na costa sudeste e sul do Brasil, para os anos (a) 2000, (b) 2001 e (c) 2002 
Tabela 1 - Médias da CPUE da raia-viola, para os anos 2000, 2001 e 2002, capturadas pelas frotas de arrasto duplo, arrasto de parelhas e arrasto simples de SC. Nota: Média \pm Erro padrão; (Mín-Máx); $\mathrm{n}=$ número de viagens.

\begin{tabular}{|c|c|c|c|c|c|c|c|c|}
\hline \multirow[t]{2}{*}{ Mês } & \multicolumn{3}{|c|}{ Arrasto Duplo } & \multicolumn{3}{|c|}{ Arrasto de Parelha } & \multicolumn{2}{|c|}{ Arrasto Simples } \\
\hline & 2000 & 2001 & 2002 & 2000 & 2001 & 2002 & 2001 & 2002 \\
\hline JAN & $\begin{array}{c}1,315 \pm 0,839 \\
(0,06-3,620) \\
n=4\end{array}$ & $\begin{array}{c}0,122 \pm 0,031 \\
(0,003-0,42) \\
n=14\end{array}$ & $\begin{array}{c}0,077 \pm 0,015 \\
(0,018-0,3) \\
n=18\end{array}$ & $\begin{array}{c}1,785 \pm 0,135 \\
(1,65-1,92) \\
n=2\end{array}$ & $\begin{array}{c}1,41 \pm 0,951 \\
(0,04-6,06) \\
n=6\end{array}$ & $\begin{array}{c}1,54 \pm 0,932 \\
(0,44-6,2) \\
n=6\end{array}$ & $\mathrm{n}=0$ & $\begin{array}{c}0,178 \pm 0,042 \\
(0,1-0,248) \\
n=3\end{array}$ \\
\hline FEV & $\begin{array}{c}0,494 \pm 0,15662 \\
(0,04-1,9) \\
n=14\end{array}$ & $\begin{array}{c}0,226 \pm 0,093 \\
(0,006-1) \\
n=10\end{array}$ & $\begin{array}{c}0,066 \pm 0,022 \\
(0,007-0,2) \\
n=9\end{array}$ & $\begin{array}{c}1,576 \pm 0,466 \\
(0,22-4,56) \\
n=9\end{array}$ & $\begin{array}{c}0,865 \pm 0,296 \\
(0,02-2,64) \\
n=10\end{array}$ & $\begin{array}{c}1,133 \pm 0,354 \\
(0,38-2,78) \\
n=6\end{array}$ & $\mathrm{n}=0$ & $\begin{array}{c}0,1 \pm 0,04 \\
(0,06-0,14) \\
n=2\end{array}$ \\
\hline MAR & $\begin{array}{c}0,402 \pm 0,1093 \\
(0,4025-0,01) \\
n=18\end{array}$ & $\begin{array}{c}0,274 \pm 0,061 \\
(0,008-0,98) \\
n=22\end{array}$ & $\begin{array}{c}0,314 \pm 0,196 \\
(0,04-1,28) \\
n=6\end{array}$ & $\begin{array}{c}1,84 \pm 0,666 \\
(0,46-3,44) \\
n=4\end{array}$ & $\begin{array}{c}2,106 \pm 0,467 \\
(0,02-5,74) \\
n=12\end{array}$ & $\begin{array}{c}1,31 \pm 0,474 \\
(0,12-3,76) \\
n=8\end{array}$ & $\mathrm{n}=0$ & $\begin{array}{c}0,109 \pm 0,037 \\
(0,04-0,167) \\
n=3\end{array}$ \\
\hline ABR & $\begin{array}{c}0,6175 \pm 0,115 \\
(0,02-1,44) \\
n=16\end{array}$ & $\begin{array}{c}0,216 \pm 0,041 \\
(0,013-0,6) \\
n=20\end{array}$ & $\begin{array}{c}0,529 \pm 0,101 \\
(0,02-1,525) \\
n=21\end{array}$ & $\begin{array}{c}0,718 \pm 0,306 \\
(0,02-1,82) \\
n=6\end{array}$ & $\begin{array}{c}0,67 \pm 0,148 \\
(0,148-1,94) \\
n=16\end{array}$ & $\begin{array}{c}0,608 \pm 0,167 \\
(0,15-1,36) \\
n=8\end{array}$ & $n=1$ & $\begin{array}{c}0,925 \pm 0,158 \\
(0,572-1,44) \\
n=5\end{array}$ \\
\hline MAI & $\begin{array}{c}0,406 \pm 0,127 \\
(0,01-1,9) \\
n=16\end{array}$ & $\begin{array}{c}0,329 \pm 0,087 \\
(0,009-1,04) \\
n=13\end{array}$ & $\begin{array}{c}0,514 \pm 0,092 \\
(0,02-2) \\
n=25\end{array}$ & $\begin{array}{c}0,421 \pm 0,182 \\
(0,041,81) \\
n=9-\end{array}$ & $\begin{array}{c}0,428 \pm 0,162 \\
(0,02-1,56) \\
n=9\end{array}$ & $\begin{array}{c}1,522 \pm 0,605 \\
(0,02-7,5) \\
n=12\end{array}$ & $n=0$ & $\begin{array}{c}0,262 \pm 0,09 \\
(0,02-0,56) \\
n=6\end{array}$ \\
\hline JUN & $\begin{array}{c}0,320 \pm 0,126 \\
(0,01-1,46) \\
n=13\end{array}$ & $\begin{array}{c}92 \pm 0,081 \\
(0,01-1,666) \\
n=25\end{array}$ & $\begin{array}{c}17,67 \pm 17,10 \\
(0,02-240) \\
n=14\end{array}$ & $\begin{array}{c}0,768 \pm 0,328 \\
(0,16-1,6) \\
n=5\end{array}$ & $\begin{array}{c}0,171 \pm 0,069 \\
(0,04-0,42) \\
n=6\end{array}$ & $\begin{array}{c}1,22 \pm 0,32 \\
(0,44-2) \\
n=4\end{array}$ & $n=1$ & $\begin{array}{c}2,136 \pm 0,912 \\
(0,12-5,42) \\
n=5\end{array}$ \\
\hline JUL & $\begin{array}{c}0,115 \pm 0,041 \\
(0,02-0,235) \\
n=5\end{array}$ & $\begin{array}{c}87,31 \pm 53,37 \\
(0,02-1,180) \\
n=26\end{array}$ & $\begin{array}{c}0,263 \pm 0,078 \\
(0,008-2,131) \\
n=30\end{array}$ & $\begin{array}{c}0,496 \pm 0,187 \\
(0,1-0,87) \\
n=4\end{array}$ & $\begin{array}{c}0,511 \pm 0,223 \\
(0,04-1,72) \\
n=9\end{array}$ & $\begin{array}{c}0,517 \pm 0,357 \\
(0,02-3) \\
n=18\end{array}$ & $n=0$ & $\begin{array}{c}0,584 \pm 0,268 \\
(0,06-1,54) \\
n=5\end{array}$ \\
\hline AGO & $\begin{array}{c}0,208 \pm 0,043 \\
(0,008-0,6) \\
n=18\end{array}$ & $\begin{array}{c}40,60 \pm 23,87 \\
(0,012-4,04) \\
n=21\end{array}$ & $\begin{array}{c}0,107 \pm 0,022 \\
(0,012-0,3) \\
n=22\end{array}$ & $\begin{array}{c}0,794 \pm 0,411 \\
(0,02-2,94) \\
n=7\end{array}$ & $\begin{array}{c}0,762 \pm 0,343 \\
(0,02-3,02) \\
n=9\end{array}$ & $\begin{array}{c}0,265 \pm 0,096 \\
(0,02-1) \\
n=10\end{array}$ & $\begin{array}{c}1,626 \pm 0,407 \\
(1,018-2,4) \\
n=3\end{array}$ & $\begin{array}{c}0,068 \pm 0,013 \\
(0,02-0,1) \\
n=5\end{array}$ \\
\hline SET & $\begin{array}{c}0,063 \pm 0,021 \\
(0,012-0,18) \\
n=10\end{array}$ & $\begin{array}{c}0,292 \pm 0,179 \\
(0,012-3,62) \\
n=20\end{array}$ & $\begin{array}{c}0,081 \pm 0,019 \\
(0,02-0,32) \\
n=16\end{array}$ & $\begin{array}{c}0,63 \pm 0,378 \\
(0,04-1,74) \\
n=4\end{array}$ & $\begin{array}{c}0,87 \pm 0,372 \\
(0,04-3) \\
n=12\end{array}$ & $\begin{array}{c}10,28 \pm 9,962 \\
(0,02-8) \\
n=8\end{array}$ & $\begin{array}{c}2,183 \pm 0,75 \\
(0,66-5,58) \\
n=6\end{array}$ & $\begin{array}{c}0,122 \pm 0,088 \\
(0,028-0,3) \\
n=3\end{array}$ \\
\hline OUT & $\begin{array}{c}0,04 \pm 0,005 \\
(0,015-0,092) \\
n=18\end{array}$ & $\begin{array}{c}0,491 \pm 0,378 \\
(0,007-4,24) \\
n=11\end{array}$ & $\begin{array}{c}0,172 \pm 0,055 \\
(0,014-0,893) \\
n=22\end{array}$ & $\begin{array}{c}0,37 \pm 0,218 \\
(0,08-1,02) \\
n=4\end{array}$ & $\begin{array}{c}0,769 \pm 0,284 \\
(0,04-3) \\
n=12\end{array}$ & $\begin{array}{c}0,192 \pm 0,054 \\
(0,02-0,7) \\
n=13\end{array}$ & $\begin{array}{c}1,265 \pm 0,749 \\
(0,02-3,22) \\
n=4\end{array}$ & $\begin{array}{c}0,09 \pm 0,03 \\
(0,06-0,12) \\
n=2\end{array}$ \\
\hline NOV & $\begin{array}{c}0,085 \pm 0,021 \\
(0,012-0,38) \\
n=18\end{array}$ & $\begin{array}{c}0,549 \pm 0,346 \\
(0,009-3,16) \\
n=9\end{array}$ & $\begin{array}{c}0,074 \pm 0,026 \\
(0,02-0,44) \\
n=17\end{array}$ & $\begin{array}{c}0,14 \pm 0,034 \\
(0,02-0,34) \\
n=12\end{array}$ & $\begin{array}{c}1,146 \pm 0,390 \\
(0,14-3,6) \\
n=8\end{array}$ & $\begin{array}{c}0,437 \pm 0,254 \\
(0,02-1,92) \\
n=7\end{array}$ & $\begin{array}{c}0,16 \pm 0,052 \\
(0,06-0,24) \\
n=3\end{array}$ & $\begin{array}{c}1,037 \pm 0,859 \\
(0,06-2,751) \\
n=3\end{array}$ \\
\hline DEZ & $\begin{array}{c}66,66 \pm 66,44 \\
(0,015-2060) \\
n=31\end{array}$ & $\begin{array}{c}0,097 \pm 0,025 \\
(0,012-0,38) \\
n=19\end{array}$ & $\begin{array}{c}0,084 \pm 0,018 \\
(0,013-0,28) \\
n=14\end{array}$ & $\begin{array}{c}1,373 \pm 0,876 \\
(0,08-9,923) \\
n=11\end{array}$ & $\begin{array}{c}0,405 \pm 0,103 \\
(0,04-1,454) \\
n=13\end{array}$ & $\begin{array}{c}0,235 \pm 0,059 \\
(0,06-0,7) \\
n=10\end{array}$ & $\begin{array}{c}0,086 \pm 0,024 \\
(0,04-0,12) \\
n=3\end{array}$ & $\mathrm{n}=1$ \\
\hline
\end{tabular}


rante o ano de 2000, onde verificou-se uma CPUE anual de 0,39 t/viagem de raia-viola, com valores mensais entre 0,13 e 1,16 t/viagem (Fig. 2a; Tab. 1), esta última alcançada em dezembro. Para o ano de 2001, um total de 194 viagens foi analisado, resultando em uma CPUE de 0,53 t/viagem (Fig. 2b; Tab. 1). Os meses de março e novembro foram os que apresentaram maiores CPUE, alcançando 1,3 e 1,15 t/ viagem, respectivamente. Em 2002, para as 131 viagens estudadas, foi observada uma CPUE anual de $0,7 \mathrm{t} /$ viagem de viola, enquanto a variação mensal foi de 0,25 a 1,3 t/viagem (Fig. 2c; Tab. 1), com CPUE mais elevadas entre janeiro e maio.

O primeiro registro de desembarque da raia-viola pela frota de arrasto simples em Santa Catarina ocorreu em abril de 2001, sendo analisadas neste ano 42 viagens de pesca, apresentando uma CPUE de 0,63 t/viagem, sendo que o mês de setembro apresentou a maior CPUE, com 1,45 t/viagem (Fig.3a; Tab. 1). $O$ ano de 2002, para as 65 viagens investigadas, revelou uma CPUE anual de $1 \mathrm{t} /$ viagem. Durante esse ano, houve um aumento gradual de CPUE até novembro, quando 3,7 t/ viagem foram desembarcadas (Fig. 3b; Tab. 1).

Um padrão de variação sazonal na CPUE da raia-viola capturada pelas três artes de pesca não foi observado, bem como uma relação com o comportamento da CPUE da captura total (Fig. 1, 2 e 3). Entretanto, foram observados picos de primavera (exceto para o ano de 2002) para capturas com arrasto duplo e de parelhas, e picos de verão para capturas com arrasto de parelhas em 2001 e 2002. No período analisado, i.e. 2000 a 2002 , verifica-se também uma tendência de aumento da CPUE, o que poderia estar associada a um aumento da disponibilidade dessas espécies quando comparado a década de 1990 (Fig. 4).

\section{Distribuição sazonal e espacial da captura}

Nas Figuras 5, 6 e 7 é apresentado a distribuição sazonal e espacial da CPUE para diferentes frotas. As capturas de raia-viola rea- lizadas pela frota de arrasto duplo se concentraram entre a região de Imbituba (SC) e Rio Grande (RS) durante o período analisado, ocorrendo maiores capturas próximo a Mostardas
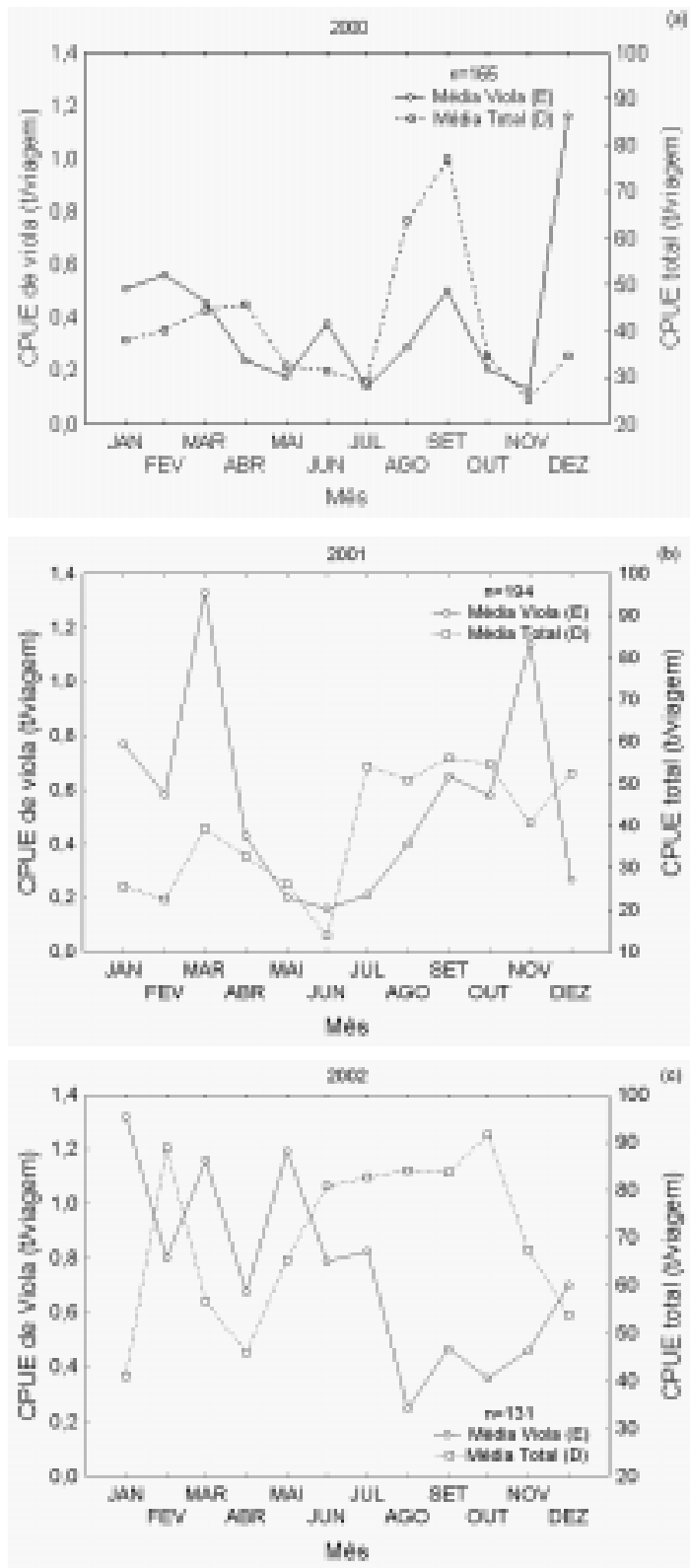

Figura 2a-c - Sazonalidade da CPUE da raia viola (Rhinobatos percellens e $R$. horkelli) pela frota de arrasto de parelha na costa sudeste e sul do Brasil, para os anos (a) 2000, (b) 2001 e (c) 2002 

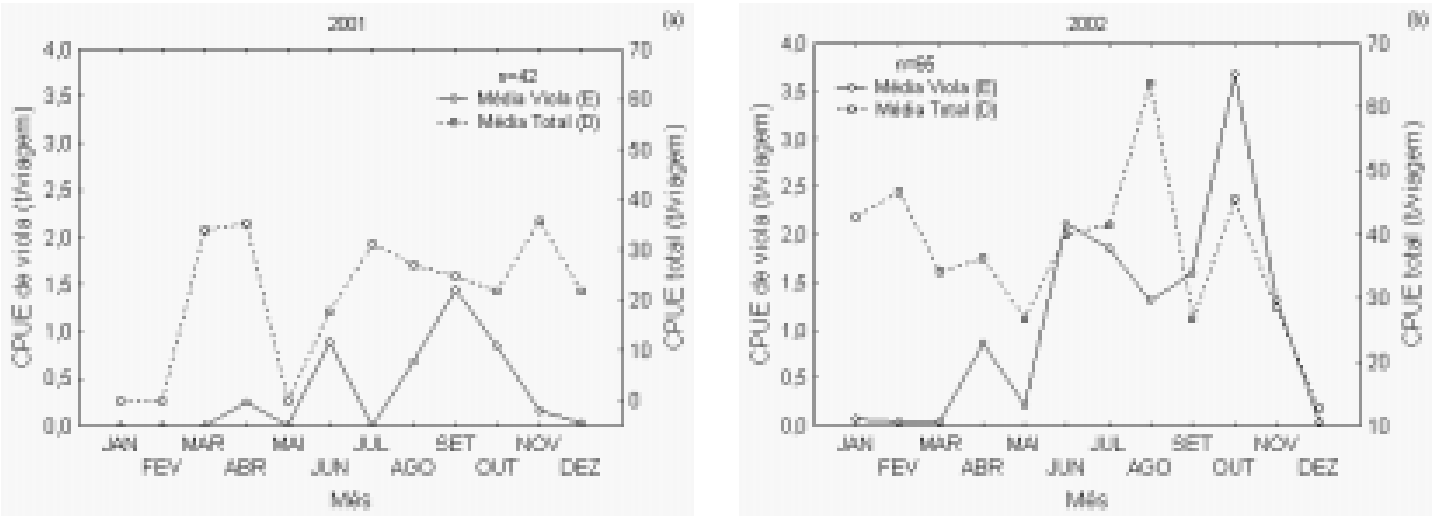

Figura 3a-b - Sazonalidade da CPUE da raia viola (Rhinobatos percellens e R. horkelli) capturada pela frota de arrasto simples na costa sudeste e sul do Brasil, para os anos (a) 2000 e (b) 2001.

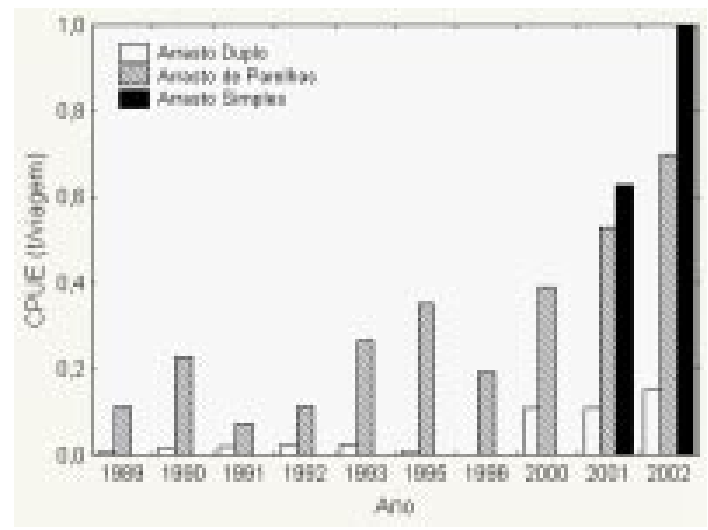

Figura 4 - Variação interanual de CPUE (t/viagem) de raia-viola desembarcadas pelas frotas de arrasto em Santa Catarina entre 1989 e 2002. Fonte: entre 1989 a 1996, IBAMA/CEPSUL (1994ab; 1998); e entre 2000 e 2002, o presente trabalho.

(RS). Ocasionalmente, capturas significativas foram registradas nos meses de inverno e verão na região do Chuí (RS). De maneira geral, observa-se que no ano 2000 as capturas da frota de arrasto duplo eram realizadas nas zonas rasas da plataforma, sendo que pescarias na zona média foram observadas apenas no outono. Os valores mais altos de CPUE não superaram as $0,68 \mathrm{t} /$ viagem neste ano. A partir de 2001, a frota passa atuar em zonas médias e profundas, as quais estão associadas a CPUE acima de 0,5 t/viagem, indicando um aumento da disponibilidade da raia-viola em maiores profundidades.

A frota de arrasto de parelhas atuou de forma tradicional durante o período estudado, ou seja, operando nas zonas rasas e raramente em zonas médias (primavera de 2001). A área onde se observa captura de viola por essa frota vai desde Tramandaí (RS) ao Chuí, ocorrendo com baixa freqüência ao norte desta região. Os dados sugerem um aumento da disponibilidade deste recurso nos meses de primavera na região costeira do Rio Grande do Sul, possivelmente relacionado com migrações das espécies para a reprodução em águas rasas neste período (Lessa et al., 1986).

Os arrasteiros simples passaram desembarcar raias-viola nos portos de Itajaí e Navegantes a partir do outono de 2001. Essas capturas são provenientes, principalmente, das zonas médias e profundas, caracterizando-se como a frota que atua mais distante da costa. As capturas da viola concentraram-se entre Imbituba e Rio Grande nos meses de outono até a primavera, sendo que freqüentemente observa-se CPUE maiores que $1 \mathrm{t}$ /viagem. Esse fato, associado às variações na CPUE dos arrasteiros duplos, indica que a raia-viola apresenta-se mais disponível em zonas acima dos 


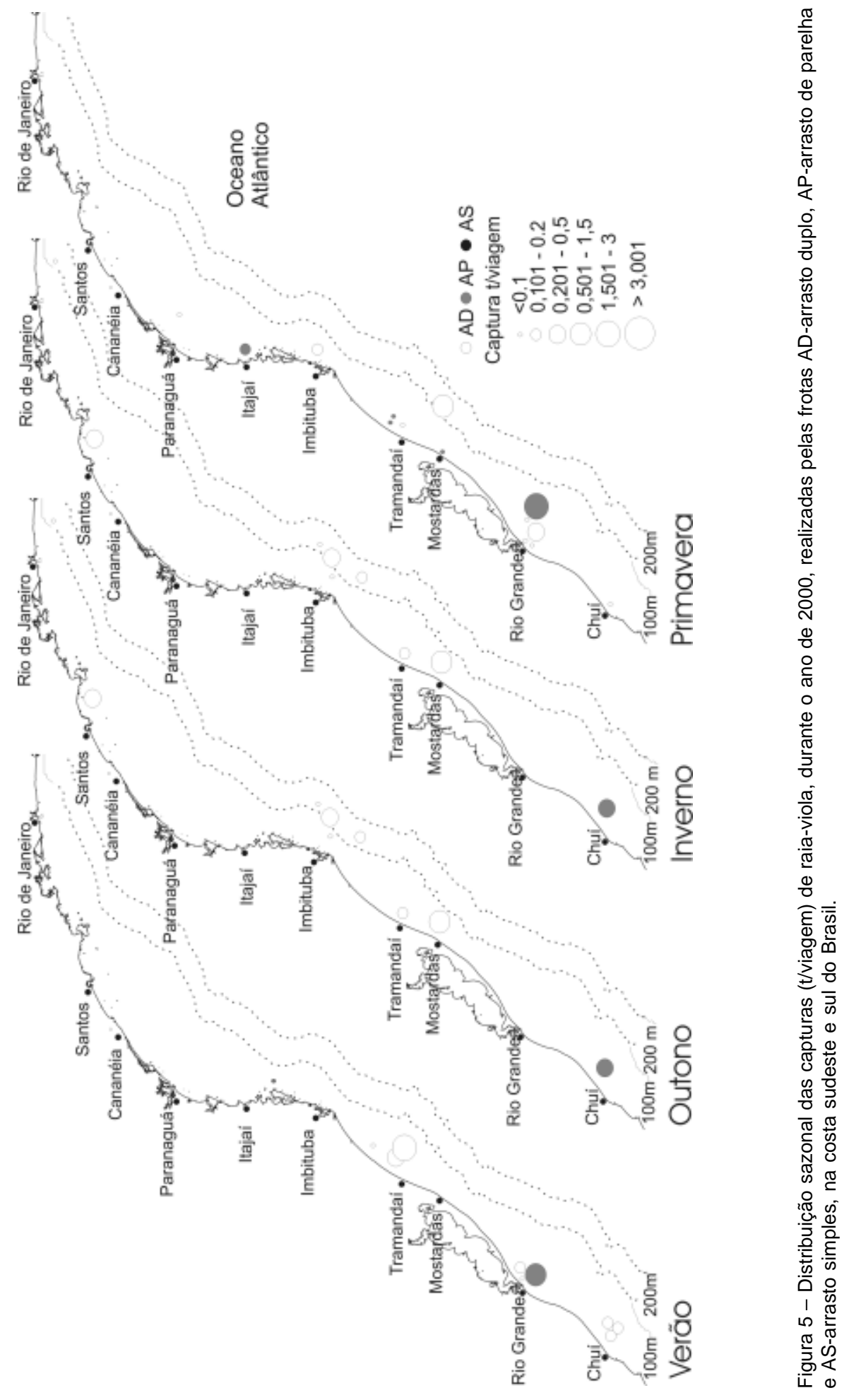




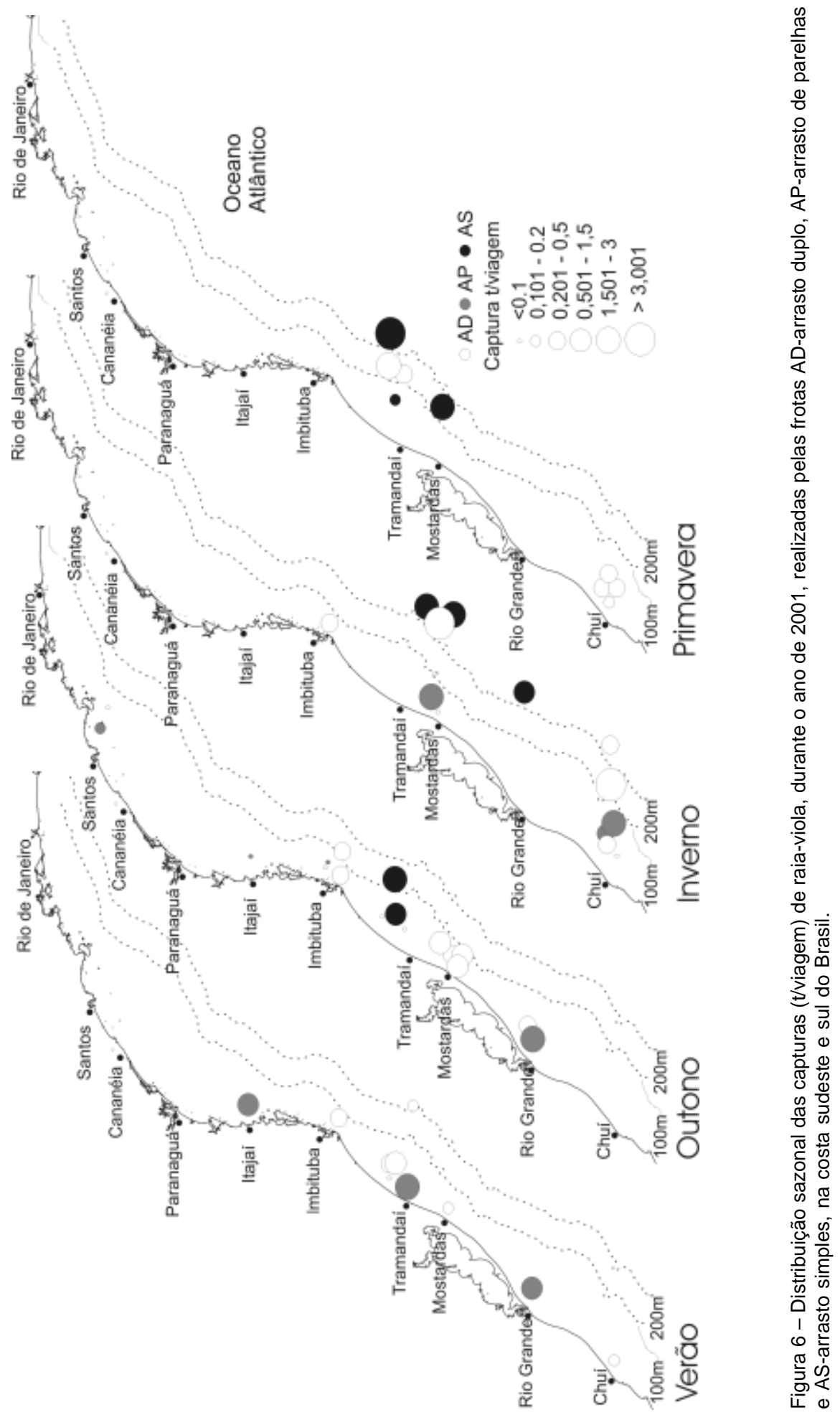




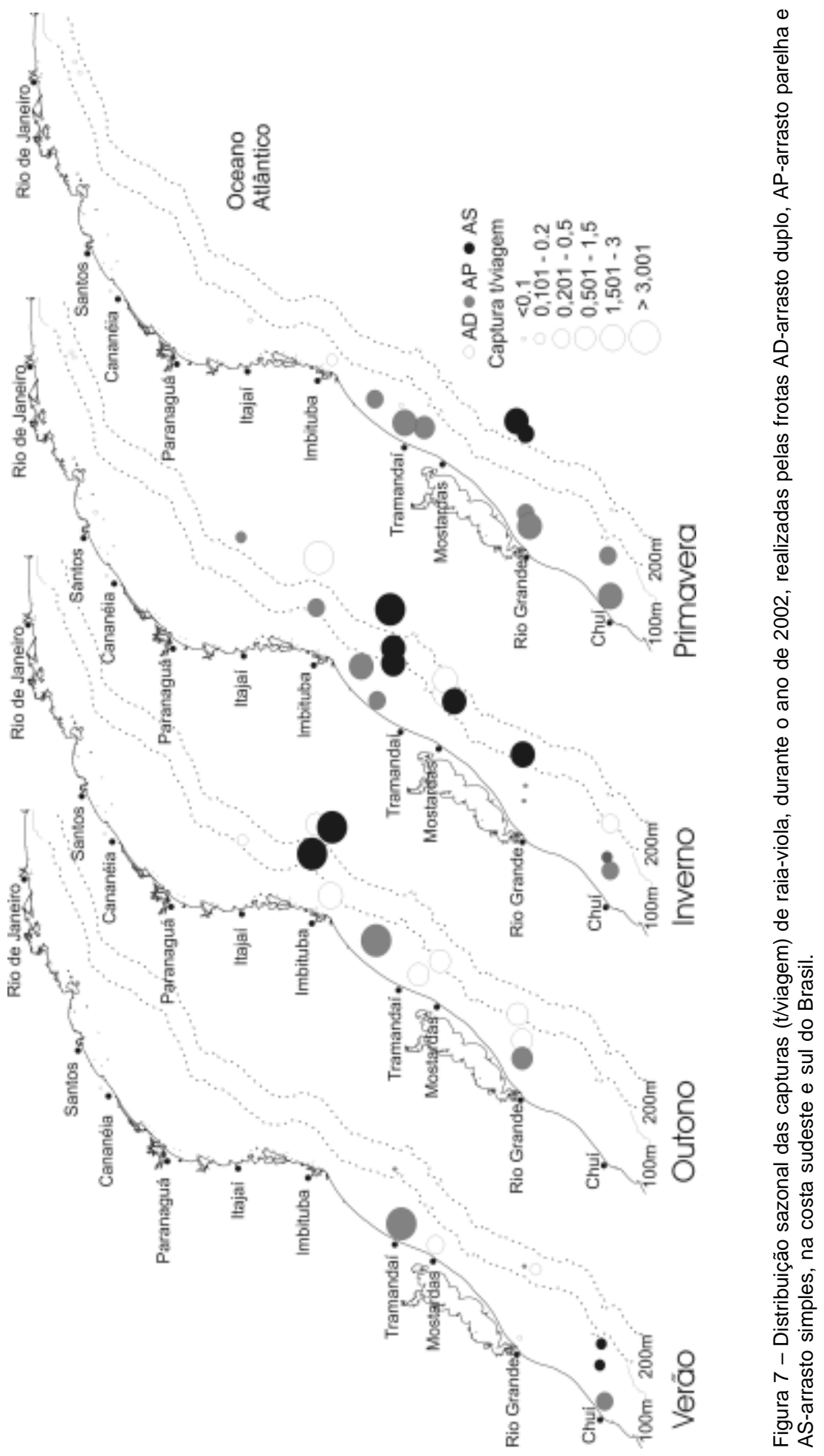


$100 \mathrm{~m}$ de profundidade. Devido à frota de arrasto de parelha atuar principalmente em zonas rasas, não se verifica um padrão de variação da CPUE com a profundidade (Fig. 8).
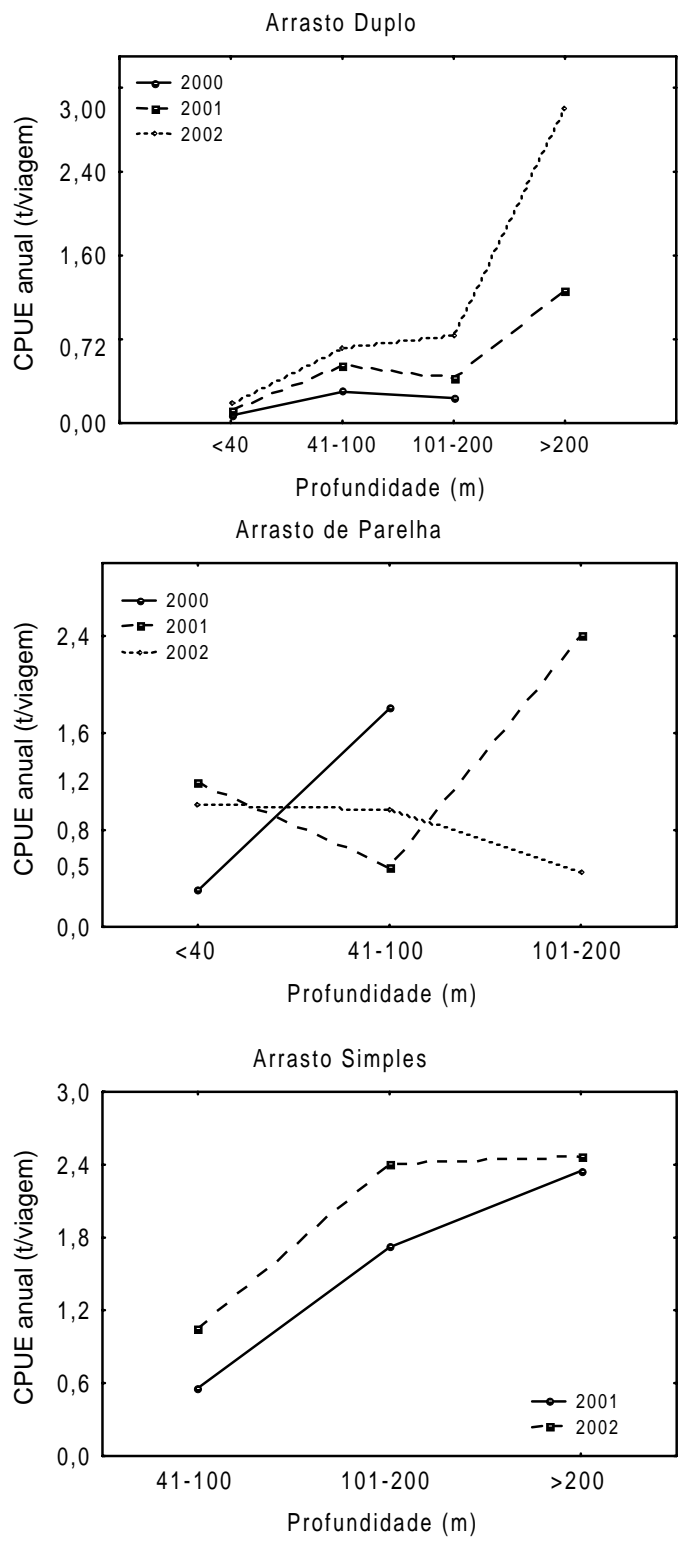

Figura 8 - Distribuição da CPUE média da raia-viola por profundidade (m) para os anos de 2000 a 2002, realizadas pelas frotas de Arrasto Duplo, Arrasto de Parelhas e Arrasto Simples, na costa sudeste e sul do Brasil.
Segundo Lessa et al. (1986), fêmeas grávidas de $R$. horkellisão abundantes em zonas rasas de novembro a março na costa do Rio Grande do Sul, quando ocorre o parto. Depois da chegada dos machos para a cópula, esses indivíduos migram novamente para zonas profundas. Considerando que a frota de arrasto duplo não operava em zonas profundas até $\mathrm{o}$ ano de 2000 e que a frota de arrasto simples começou atuar a partir de 2001 , as águas com profundidades acima de $100 \mathrm{~m}$ preservavam indivíduos da atividade pesqueira até este período. Desta forma, essa parcela protegida da população poderia representar uma reserva de biomassa para recompor os efeitos da mortalidade por pesca nas zonas rasas.

O deslocamento das frotas de arrasto para zonas profundas explica o aumento de CPUE para a raia-viola nos desembarques em Santa Catarina entre 2000 e 2002, especialmente, quando comparadas às drásticas quedas de CPUE ocorridas nos anos de 1990, na costa sudeste e sul do Brasil, que colocavam a espécie como colapsada para a pesca (Vooren, 1998; Perez et al., 2001).

\section{CONCLUSÃO}

O panorama revelado no presente estudo mostra que as frotas de arrasto do sul do Brasil passaram a atuar em zonas mais profundas, atingindo partes de estoques pesqueiros que antes eram totalmente ou parcialmente inexplorados. Desta forma, a raia-viola está submetida, atualmente, a uma pressão pesqueira que atua com maior amplitude sobre sua área de distribuição. Os aumentos de CPUE observados entre 2000 e 2002 não representam um incremento na disponibilidade da raiaviola no sudeste e sul do Brasil, mas sim, um reflexo das mudanças de estratégia de operação das frotas de arrasto na região.

Este deslocamento da frota pode provocar uma acentuada queda na CPUE para a raiaviola nos próximos anos, ainda mais, considerando que $58 \%$ dos indivíduos capturados em 
2001 e 2002 são juvenis (Martins, 2003). O ano de 2003 já revela uma queda da produção catarinense de raia-viola em mais de $50 \%$ em relação a 2002, tendo passado de 428 t para cerca de 200 t (UNIVALI/CTTMar, 2003). Avaliando-se este cenário, faz-se necessária a inclusão das espécies do gênero Rhinobatos em um futuro ordenamento da pesca multiespecífica profunda no sudeste e sul do Brasil.

\section{AGRADECIMENTOS}

Os autores gostariam se agradecer ao CTTMar/UNIVALI pelo apoio e logística durante a realização deste trabalho.

\section{REFERÊNCIAS BIBLIOGRÁFICAS}

Figueiredo, J.L. 1977. Manual de peixes marinhos do sudoeste do Brasil. São Paulo: Ed. Museu de Zoologia da Universidade de São Paulo.

Haimovici, C.M.; Castello, J.P \& C.M. Vooren. 1998. Pescarias. In: Seelinger, U.; Odebrecht, C.; Castello, J.P, Os ecossistemas costeiro e Marinho do extremo sul do Brasil. Rio Grande: Ed. Ecoscientia.

IBAMA/CEPSUL. 1994a. Desembarques controlados de pescados no Estado de Santa Catarina, 1993. Itajaí.

IBAMA/CEPSUL. 1994b. Informe sobre os desembarques controlados de pescados no Estado de Santa Catarina, nos anos de 1988 a 1992. Itajaí.

IBAMA/CEPSUL. 1998. Informe da Pesca Extrativista Marinha em Santa Catarina 1995 e 1996. Itajaí.

Lessa, R; Vooren, C.M \& Lahaye, J. 1986. Desenvolvimento e ciclo reprodutivo das fêmeas, migrações e fecundidade da viola Rhinobatos horkelli (Muller \& Henle, 1841) do sul do Brasil. Atlântica, 8: 5-34.
Martins, R.R.M. 2003. Abundância relativa do gênero Rhinobatos (Rajiformes, Rhinobatidae) na costa sudeste e sul do Brasil. Trabalho de Monografia, PUC-PR.

Mazzoleni, R.C. \& P.R Schwingel. 1999. Elasmobranch species landed in Itajaí harbor, southern Brazil. Notas Téc. FACIMAR, 3: 1-34.

Perez, J.A.A.; Pezzuto, P.R.; Rodrigues, L.F.; Valentini, H. \& C.M. Vooren. 2001. Relatório da reunião técnica de ordenamento da pesca de arrasto nas regiões sudeste e sul do Brasil - Ano 2000. Notas Téc. FACIMAR, 5: 1-34.

Pezzuto, P.R.; Andrade, H.A.; Perez, J.A.A. \& P.R. Schwingel. 2001. Boletim Estatístico da Pesca Industrial de Santa Catarina. Itajaí: Ed. da Universidade do Vale do Itajaí.

UNIVALI/CTTMar. 2003. Produção Pesqueira de Santa Catarina on-line. Disponível em: www.gep.cttmar.univali.br. Acesso em: 18 dez, 2003.

Vooren, C.M. 1998. Elasmobrânquios demersais. p.157-162. In: Seelinger, U.; Odebrecht, C.; Castello, J.P., Os ecossistemas costeiro e Marinho do extremo sul do Brasil. Rio Grande: Ed. Ecoscientia. 